\title{
Supervised Brain Emotional Learning
}

\author{
Ehsan Lotfi \\ Department of Computer Engineering, \\ Torbet-e-Jam Branch, Islamic Azad University, \\ Torbat-e-Jam, Iran \\ elotfi@ieee.org, esilotf@,gmail.com
}

\author{
M.-R. Akbarzadeh-T \\ Departments of Electrical Engineering and Computer \\ Engineering Ferdowsi University of Mashhad, Iran and \\ IEEE Senior Member \\ akbarzadeh@ieee.org
}

\begin{abstract}
In this paper we propose the supervised version of neuro-based computational model of brain emotional learning (BEL). In mammalian brain, the limbic system processes emotional stimulus and consists of following two main components: amygdala and orbitofrontal cortex (OFC). Recently several models of BEL based on monotonic reinforcement learning in amygdala are proposed by researchers. Here, we introduce supervised version of BEL which can be learned by pattern-target examples. According to the experimental studies, where various comparisons are made between the proposed method, multilayer perceptron (MLP) and adaptive neuro-fuzzy inference system (ANFIS), the main feature of the presented method is fast training in prediction problems.
\end{abstract} indix

Keywords- BELBIC; supervised learning; limbic; geomagnetic

\section{INTRODUCTION}

Emotions are cognitive processes and multidisciplinary studies of emotion have a long history. From the psychological point of view, emotions can be derived by reward and punishment received from various real-life situations and studies of the neural basis of emotion are described using the limbic system (LS) [1-2]. The LS processes the emotional stimuli associated with reward or punishment [3-4] and is located in the cerebral cortex and consists of the following components [4]: amygdala, orbitofrontal cortex (OFC), thalamus, sensory cortex, hypothalamus and hippocampus (Fig. 1). Amygdala is located in sub-cortical area and its main cognitive functions are long term memory and responsibility for emotional stimuli [4-5]. Amygdala receives connections from the sensory cortical areas [4-5] and also interacts with the OFC. OFC tries to prevent inappropriate responses from the amygdala based on the context provided by the hippocampus [6].

Recently, researchers in artificial intelligence try to present computational models of LS. Morén and Balkenius [46] proposed a neuropsychological motivated computational model of the amygdala-orbitofrontal system. The main feature of the amygdala-orbitofrontal system is that the weights of amygdala cannot decrease (called monotonic learning). So once an emotional reaction is learned, it is permanent and cannot be unlearned. In this model, the reward signal is not clearly defined and this signal is vital for updating the learning weights of system. Lucas et al. [7] explicitly determined the reward as reinforcer signal and proposed the BEL base controller (BELBIC) which has been successfully utilized in various control applications [8-18]. BELBIC is an action generation mechanism based on sensory inputs and emotional cues. Also BEL based on reinforcement learning was proposed to predict the $K p$ index of geomagnetic activity [19]. Babaei et al. [20] formulized the input reinforcer for multi agent optimization problems and presented a BEL based predictor to forecast $A E$ index in alarm systems for satellites. The $K p, A E$ and $D s t$ indices characterize the solar winds and geomagnetic storms that is a complex system and can greatly disturb communication systems and damage satellites [20-21]. These indices have chaotic behaviour and can be considered as time series. And researchers use them as case study to evaluate their methods [20-21]. The high values of $K p$ and $A E$ and the large variation at low values of Dst often correspond to geomagnetic storms or sub storms [22-26].

All reviewed BEL models are based on monotonic reinforcement learning and need an input reward extracted from input data. Also all reviewed BEL based predictors show high accuracy in predicting peak points but not at the all points [20] particularly when signal level is low. They show low accuracy at low points. So they cannot predict some time series such as Dst index where the low values are most important. Here, we proposed supervised version of BEL that can be learned by using pattern-target examples. The proposed method can be used to predict the Dst index along with other indices such as $K p$ and $A E$ as case study.

The organization of the paper is as follows: The proposed method is illustrated in Section 2. Section 3 presents the comparison between proposed method, multilayer perceptron (MLP) and adaptive neuro-fuzzy inference system (ANFIS) [27] which are popular predictors in geomagnetic phenomena forecasting.

\section{PROPOSED SUPERVISED BEL}

In contrast to previous methods, proposed method can be learned by pattern-target examples. Actually the supervised version of BEL is introduced here. The proposed method can be utilized in various prediction and fitting problems. Additionally, according to the previous section, the main 
feature of the amygdala-orbitofrontal system and its modified versions is monotonic learning. It is observed that by controlling the monotonic learning, the performance of the model can be extended. In other words, a decay mechanism is needed. The next modification introduced with respect to the basic model and its modified versions is applying a decay rate $\gamma$ in amygdala learning rule.

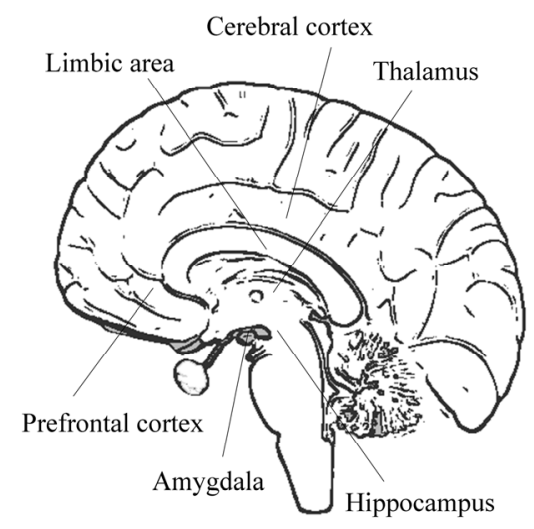

Fig. 1. The limbic system in the brain

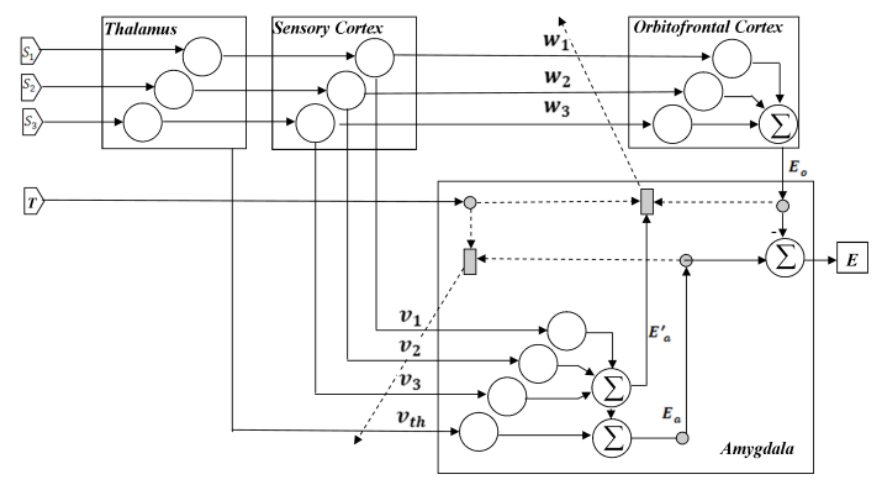

Fig. 2. Proposed computational model for BEL

The proposed model as 3 inputs single output is shown in the Fig. 2. In Fig. 2, the solid lines present the data flow and learning lines are presented by dashed lines. The adjustable weights include $w_{1}, w_{2}, w_{3}, v_{l}, v_{2}, v_{3}$ and $v_{t h}$. And the proposed supervised learning rules are as follows:

$$
\begin{gathered}
v_{j}^{k+1}=(1-\gamma) v_{j}^{k}+\alpha \max \left(T^{k}-E_{a}^{k}, 0\right) s_{j}^{k} \\
v_{t h}^{k+1}=(1-\gamma) v_{t h}^{k}+\alpha \max \left(T^{k}-E_{a}^{k}, 0\right) \cdot \max _{j}\left(s_{j}^{k}\right) \\
w_{j}^{k+1}=w_{j}^{k}+\left(\beta R_{0} s_{j}^{k}\right)
\end{gathered}
$$

Where $k$ is learning step, $\alpha$ and $\beta$ are learning rates, $\gamma$ is proposed decay rate, $s_{j}^{k}$ is $j$ th element of $k$ th sensory input, and

$$
E_{a}^{k}=\sum_{j, j \neq t h}\left(v_{j}^{k} s_{j}^{k}\right)
$$

$T^{k}$ is the target value associated with $k$ th sensory input $s^{k}$, and $R_{0}$ is the internal reward calculated by the following formula:

$$
R_{0}=\left\{\begin{array}{cc}
\max \left(E_{a}^{\prime k}-T^{k}, 0\right)-E_{o}^{k} & \text { if }\left(T^{k} \neq 0\right) \\
\max \left(E_{a}^{\prime k}-E_{o}^{k}, 0\right) & \text { Otherwise }
\end{array}\right.
$$

Where,

$$
\begin{aligned}
E_{a}^{\prime k} & =\sum_{j, j \neq t h}\left(v_{j}^{k} s_{j}^{k}\right) \\
E_{o}^{k} & =\sum_{j}\left(w_{j}^{k} s_{j}^{k}\right)
\end{aligned}
$$

Basically the model is divided into two parts, corresponding to the amygdala and the OFC. The amygdala receives input pattern from the thalamus and from the sensory cortex, while the OFC only receives input pattern from the cortical areas. Amygdala has two internal outputs: $E_{a}$, that is used for adjusting its own weights (see Eq. 1 and Eq. 2) and $E_{\text {a }}$ that is used for adjusting OFC weights (see Eq. 3 and Eq. $5)$. Beside input pattern the model also needs a Target $(T)$ to adjust the weights. As shown in Fig. 2, the input pattern is described by the vector $s$. There is one node for each attribute of input pattern $s$ in the network model of amygdale and OFC. The output of each node is calculated by the multiplication of a plastic connection weight $v_{j}$ to $s_{j}$ for amygdala and $w_{j}$ to $s_{j}$ for OFC. In Fig. 2, the $E$ is the final output node. And $E^{k}$ is output value associated with $k$ th sensory input, $s^{k}$. It's calculated simply by the following formula:

$$
E^{k}=E_{a}^{k}+v_{t h}^{k} \times \max _{j}\left(s_{j}^{k}\right)-E_{o}^{k}
$$

Where, $k$ is learning step, $\max _{j}\left(s_{\mathrm{j}}{ }^{\mathrm{k}}\right)$ is the output of thalamus and $v_{t h}{ }^{k}$ is the related weight. In the equation, subtraction implements the inhibitory task of OFC. The model can be used in time series prediction problems. Consider the following time series:

$$
K p_{1}, K p_{2}, K p_{3}, \ldots, K p_{t}, \ldots
$$

Table 1 shows the training pattern-target pairs extracted from the time series. In the learning phase, the input pattern composes of the element of stimuli ( $s$ in Eqs. (1), (2), (3), (6) and (7)) and the value $T$ is set as target. 
TABLE 1 THE PATTERN-TARGET PAIRS EXTRACTED FROM $K P$ TIME SERIES

\begin{tabular}{c|c|c}
\hline ID & Pattern & target \\
\hline \hline$P_{1}$ & $K p_{1}, K p_{2}, K p_{3}, K p_{4}$ & $K p_{5}$ \\
$P_{2}$ & $K p_{2}, K p_{3}, K p_{4}, K p_{5}$ & $K p_{6}$ \\
$P_{3}$ & $K p_{3}, K p_{4}, K p_{5}, K p_{6}$ & $K p_{7}$ \\
$\ldots$ & $\ldots$ & $\ldots$ \\
$P_{t-5}$ & $K p_{t-4}, K p_{t-3}, K p_{t-2}, K p_{t-1}$ & $K p_{t}$ \\
& &
\end{tabular}

\section{EXPERIMENTAL STUDIES}

The $K p, A E$ and $D s$ indices are used to characterize the geomagnetic activity of the earth's magnetosphere which is a complex dynamical system. These time series have chaotic behavior. 184104 hourly samples data set from 1976 to 1996 (solar cycles 21 and 22) has been downloaded from National Space Science Data Center (NSSDC). We considered each 4 sequence samples as a pattern and 5th as its target. So 184100 pattern-target pairs of $\mathrm{Kp}$ index and 184100 pattern-target pairs of Dst index are used for the evaluations. More than $26 \%$ of $\mathrm{AE}$ data are omitted. The missed values in standard $\mathrm{AE}$ dataset were filled with 9999 and 135832 pattern-target pairs of $\mathrm{AE}$ index have been used. The maximum and minimum of each index are determined and scaled data (between 0 and 1) are used to adjust the weights.

For all learning scenarios listed below, the training set contains $70 \%$ while the testing set contains $15 \%$ of the data. Also $15 \%$ of data is used for the validation set. The stopping criterion in learning process is validation check that is as follow: "if validation performance decreased and does not show an increase for 3 consecutive epochs then stop the algorithm." Also the maximum number of learning epochs is 1000. The initialization of the weights is random and during each learning epoch, all training pattern-target pairs are applied. Finally the values $\alpha, \beta$ and $\gamma$ are set respectively at $0.8,0.2$ and 0.01 .

\section{A. Kp prediction Results}

Fig. 3 is the curves of predicted values of $K p$ versus target values in the training, validation and test sets. And Fig. 4 shows the target and predicted $K p$ values obtained from DBEL at 500 hours in year 1992. Table 2 presents the percentage recall, missed and false detection of $K p$ at high extremes. The thresholds include: predicted $K p$ values that are more than 4, 5 and 6. For example the second column of the table is calculated by following equations:

$$
\begin{aligned}
& \text { recall }(K p>4)=\frac{\text { Correctly Detected as } K p>4}{\text { Total Number Satisfied } K p>4} \times 100 \\
& \text { false }(K p>4)=\frac{\text { Incorrectly Detected as } K p>5,6}{\text { Total Number Satisfied Kp }>4} \times 100 \\
& \text { missed }(K p>4)=\frac{\text { Incorrectly Detected as } K p<4}{\text { Total Number Satisfied Kp }>4} \times 100
\end{aligned}
$$
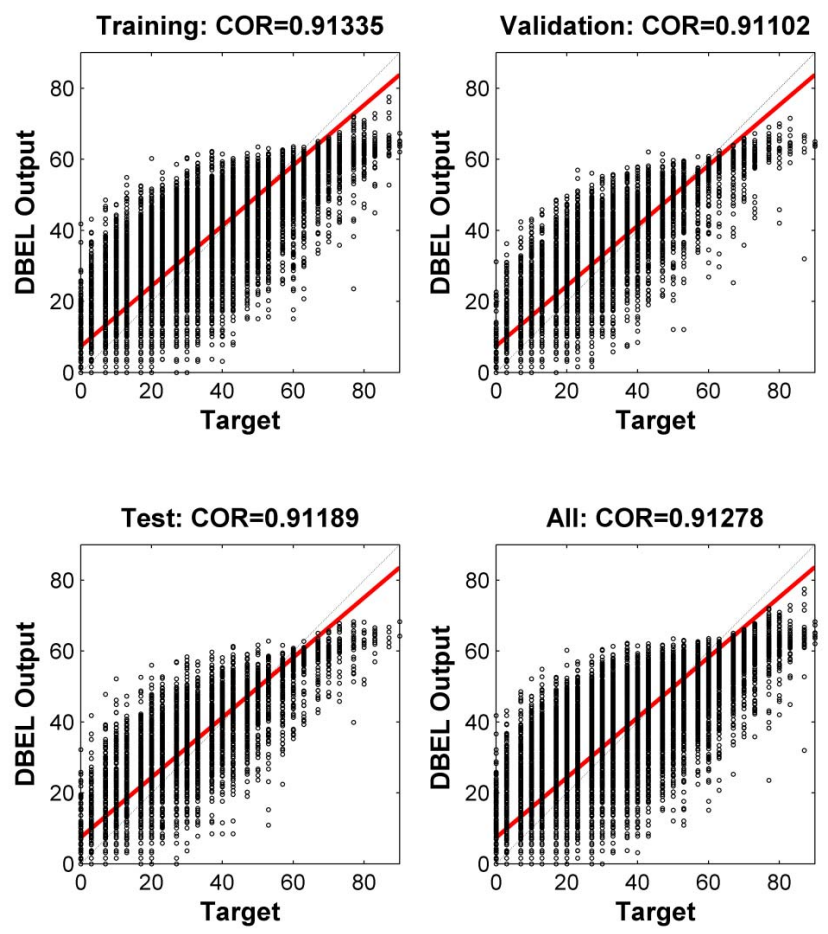

Fig. 3 Actual versus desired output of the $K p(\times 10)$ obtained from proposed method

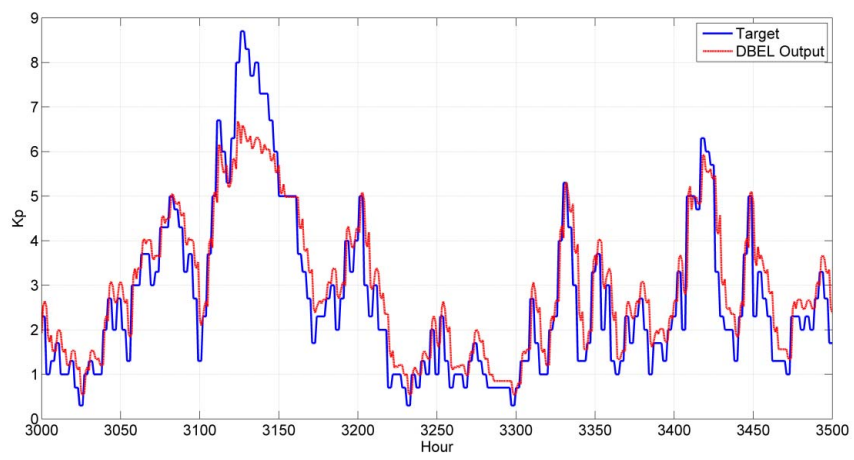

Fig. 4 Predicted $K p$ values between hours 3000-3500 in year 1992 obtained from proposed method 
TABLE 2 THE ACCURACY OF KP PREDICTIONS AT HIGH EXTREMES

\begin{tabular}{c|cccccccccc}
\hline threshold & \multicolumn{3}{|c}{$\boldsymbol{K} \boldsymbol{p}>\mathbf{4}$} & \multicolumn{3}{c}{$\boldsymbol{K} \boldsymbol{p} \mathbf{5}$} & \multicolumn{3}{c}{$\boldsymbol{K} \boldsymbol{p} \mathbf{6}$} \\
\hline Model & ANFIS & DBEL & MLP & ANFIS & DBEL & MLP & ANFIS & DBEL & MLP \\
\hline \hline recall & $84.83 \%$ & $32.90 \%$ & $70.59 \%$ & $79.71 \%$ & $74.98 \%$ & $42.25 \%$ & $69.97 \%$ & $82.54 \%$ & $23.72 \%$ \\
false & $12.85 \%$ & $65.80 \%$ & $23.09 \%$ & $12.09 \%$ & $19.22 \%$ & $38.54 \%$ & $4.86 \%$ & $4.15 \%$ & $8.07 \%$ \\
missed & $2.32 \%$ & $1.29 \%$ & $6.32 \%$ & $8.21 \%$ & $5.80 \%$ & $19.21 \%$ & $25.17 \%$ & $13.31 \%$ & $68.22 \%$
\end{tabular}

According to the Table 2, DBEL provides better results for $K p>6$. The higher ratio of recall and false detections and lower ratio of missed detection clarify that the DBEL based predictor is more sensitive than the MLP and ANFIS based predictor in high values of $K p$.

TABLE 3 COMPARISONS BETWEEN MLP, ANFIS AND DBEL IN KP AND AE PREDICTION

\begin{tabular}{c|cccccc}
\hline index & \multicolumn{5}{|c}{$\boldsymbol{K} \boldsymbol{p}$} & \multicolumn{3}{c}{$\boldsymbol{A E}$} \\
\hline Model & RMSE & COR & epoch & RMSE & COR & epoch \\
\hline \hline MLP & 2.7126 & 0.87935 & 1000 & 321.3884 & 0.9906 & 1000 \\
ANFIS & 0.5023 & 0.93803 & 60 & 116.6683 & 0.99967 & 60 \\
DBEL & 0.6838 & 0.91189 & 3 & 149.8850 & 0.99942 & 3
\end{tabular}

Finally Table 3 compares the RMSE, COR of the results obtained from DBEL, MLP and ANFIS in test set. As illustrated in Table 3, the DBEL shows an improvement with respect to the MLP. The best agreement between the predicted $K p$ values and target values is obtained from ANFIS. On the other hand, DBEL learns the $K p$ data just in three epochs while MLP and ANFIS with backpropagation algorithm need many epochs to learn.

\section{B. AE prediction results}

Also Table 3 shows the comparisons between the three methods in $A E$ prediction problem. Table 3 presents the RMSE and COR of the results in test set and illustrates the number of learning epochs for MLP, ANFIS and DBEL. Higher correlation and lower root mean square error for DBEL mean that DBEL has a better performance than MLP. According to the Table 3, DBEL just during 3 epochs reaches more than $99 \%$ of COR results which were obtained from ANFIS in $A E$ prediction. So fast training is the main feature of DBEL with respect to the ANFIS. Fig. 5 illustrates the CORs plot of $A E$ index in training, validation and test sets. And Fig. 6 shows the target and predicted $A E$ values between hours
3000-3500 in year 1992 obtained from DBEL. Table 4 presents the prediction results of the peak points. The DBEL correctly detects more that $99.86 \%$ while the ANFIS detects $10 \%$ peak points which are more than 600. Also DBEL presents better results for $A E>1000$ than ANFIS and MLP.

TABLE 6 THE DST PREDICTION RESULTS

\begin{tabular}{c|ccc}
\hline Model & RMSE & COR & epoch \\
\hline \hline MLP & 10.1044 & 0.9238 & 1000 \\
ANFIS & 4.7391 & 0.98143 & 50 \\
DBEL & 8.5084 & 0.94351 & 5
\end{tabular}
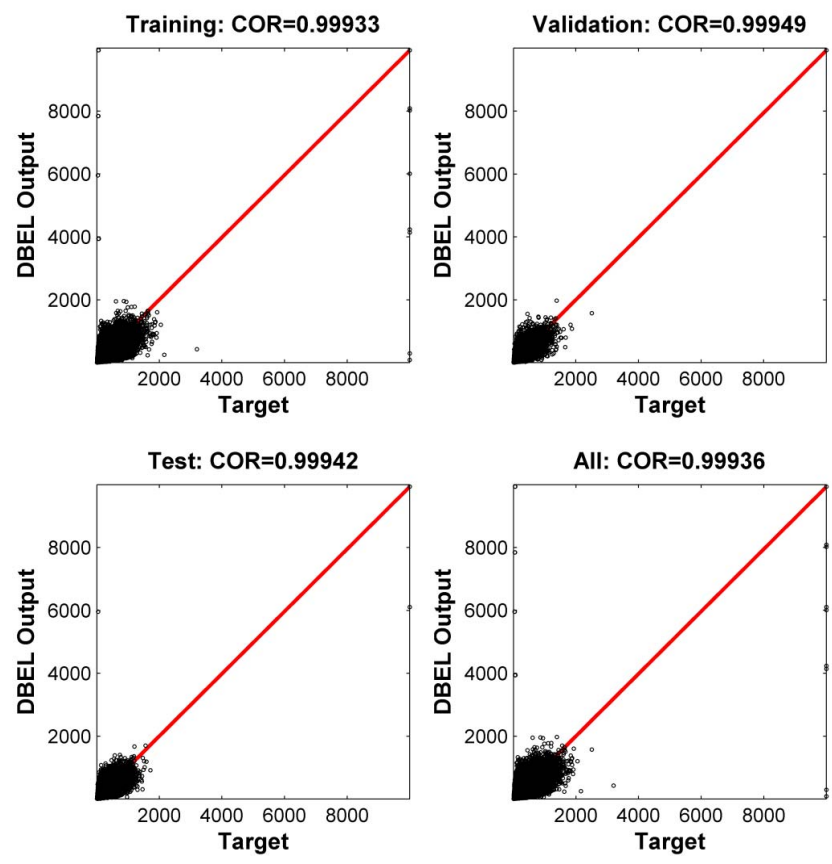

Fig. 5 Actual versus desired output of the $A E$ obtained from DBEL

TABLE 4 THE ACCURACY OF PREDICTED AE AT PEAK POINTS

\begin{tabular}{c|ccccccccc}
\hline threshold & \multicolumn{3}{|c}{ AE>600 } & \multicolumn{3}{c}{ AE>1000 } & \multicolumn{3}{c}{ AE>1400 } \\
\hline Model & ANFIS & DBEL & MLP & ANFIS & DBEL & MLP & ANFIS & DBEL & MLP \\
\hline \hline recall & $10.00 \%$ & $99.86 \%$ & $99.95 \%$ & $15.36 \%$ & $17.93 \%$ & $0.00 \%$ & $45.42 \%$ & $43.28 \%$ & $4.14 \%$ \\
false & $80.00 \%$ & 0.11 & $0.00 \%$ & $64.51 \%$ & $54.89 \%$ & $10.42 \%$ & $1.81 \%$ & $3.44 \%$ & $0.00 \%$ \\
missed & $10.00 \%$ & $0.00 \%$ & $0.00 \%$ & $20.14 \%$ & $27.17 \%$ & $89.58 \%$ & $52.78 \%$ & $53.27 \%$ & $95.86 \%$
\end{tabular}


TABLE 5 THE ACCURACY OF PREDICTED DST AT LOW POINTS

\begin{tabular}{c|cccccccccc}
\hline threshold & \multicolumn{3}{|c}{ Dst<-25 } & \multicolumn{3}{c}{ Dst $<-50$} & \multicolumn{3}{c}{ Dst $<-\mathbf{1 0 0}$} \\
\hline Model & ANFIS & DBEL & MLP & ANFIS & DBEL & MLP & ANFIS & DBEL & MLP \\
\hline \hline recall & $87.56 \%$ & $75.28 \%$ & $59.73 \%$ & $89.62 \%$ & $74.86 \%$ & $45.17 \%$ & $86.24 \%$ & $76.23 \%$ & $51.18 \%$ \\
missed & $12.44 \%$ & $5.03 \%$ & $0.85 \%$ & $10.18 \%$ & $22.39 \%$ & $53.25 \%$ & $4.21 \%$ & $22.32 \%$ & $48.82 \%$ \\
false & $0.00 \%$ & $19.69 \%$ & $39.42 \%$ & $0.19 \%$ & $2.75 \%$ & $1.58 \%$ & $9.54 \%$ & $1.45 \%$ & $0.00 \%$
\end{tabular}

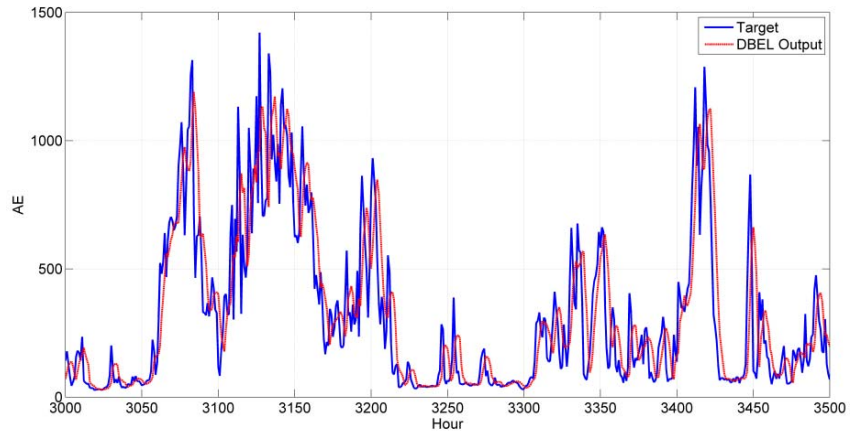

Fig. 6 Predicted $A E$ values between hours 3000-3500 in year 1992 obtained from DBEL

\section{Dst prediction results}

The prediction results of the Dst index are presented in the Table 5, Table 6, Fig. 7 and Fig. 8. The correlation plots of the results in training set, test set and validation set are separately presented in Fig. 7. The target and predicted values between hours 3000-3500 in year 1992 are illustrated in Fig. 8. The results of the predicted Dst values are based on the following three thresholds: less than -25 , less than -50 and less than -100 presented in Table 5. According to the Table 5, DBEL does more accurate predictions for low values of Dst. The recall ratio and false detection ratio for DBEL is higher than the MLP based predictor. Also the missed detection ratio of the DBEL based predictor is lower than the MLP. So DBEL is more accurate and more sensitive than the MLP based predictor for low values of Dst.

The ANFIS recall ratio is higher than DBEL result while the number of learning epochs of DBEL is much less than ANFIS. As illustrated in Table 6, after 5 epochs, RMSE = 8.5084 and $\mathrm{COR}=0.94351$ are obtained for DBEL. And they are very close to the results obtained using ANFIS after 50 epochs.
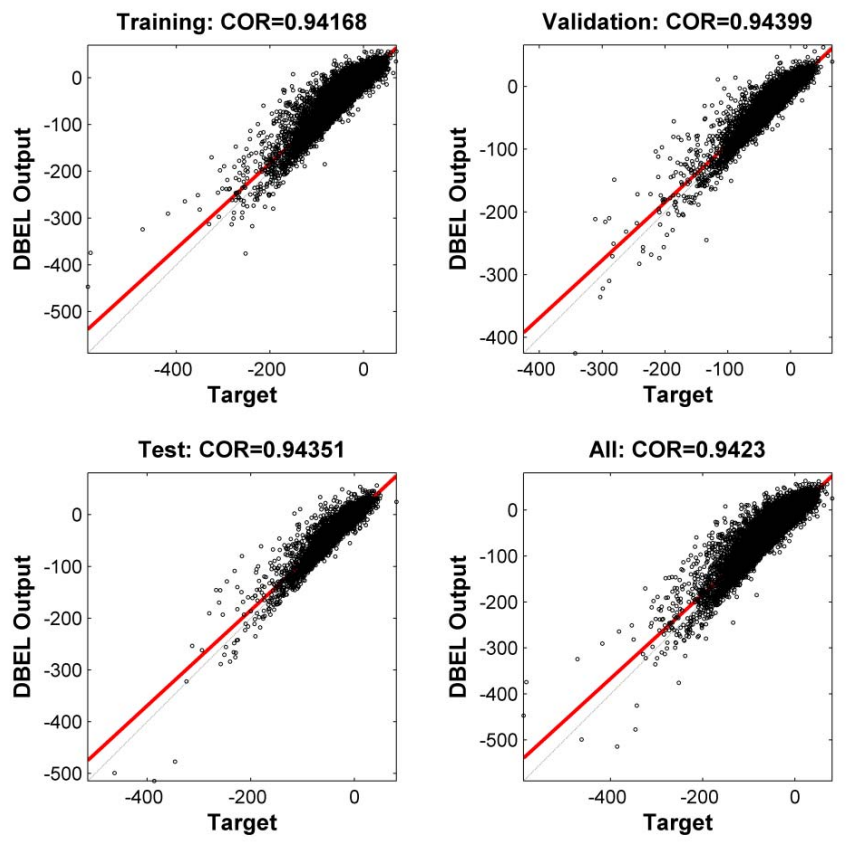

Fig. 7 Actual versus desired output of the Dst obtained from DBEL

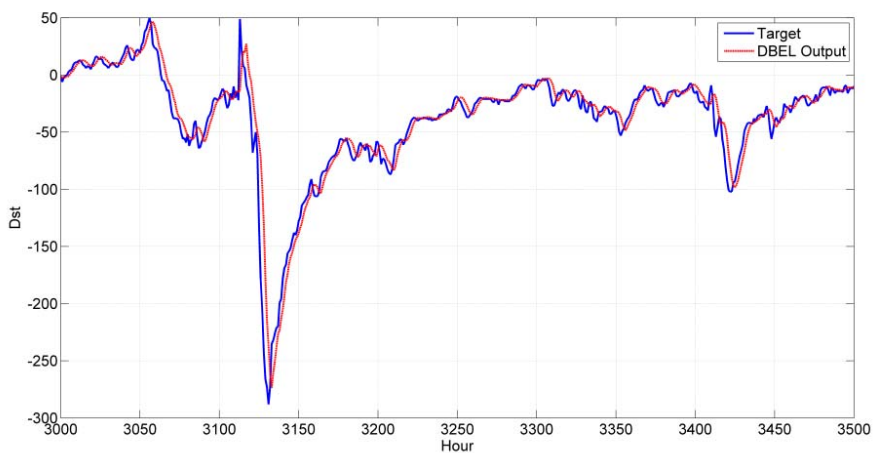

Fig. 8 Predicted $D s t$ values between hours 3000-3500 in year 1992 obtained from DBEL

\section{CONCLUSION}

In this paper we present cognitive model of the brain's emotional learning with a novel configuration. The main modification that is introduced here, with respect to the basic 
model (BEL), is applying decay rate $\gamma$ in amygdale learning rule. Also instead of reward signal, we used the target value in the learning rules. In other words, the supervised version of BEL is introduced here. The proposed model is named DBEL (supervised brain emotional decayed learning) and it was utilized to predict $K p$ and $A E$ indices, which their high values have great importance, and Dst of geomagnetic index, which its low values involve critical areas. According to the experimental studies, comparisons between DBEL, MLP and ANFIS in $K p, A E$ and $D s t$ offline prediction present the following conclusions: First, the performance of DBEL is higher than MLP especially at high and low values and is higher than ANFIS at high extremes. Second, DBEL just during 3 till 5 epochs reaches more than $99 \%$ of COR results which were obtained using ANFIS at all points. Third, lower computational complexity and faster training for DBEL make it suitable for real time predicting systems. DBEL is based on neurophysiological aspect of the brain emotional learning with a very simple structure. DBEL is novel supervised learning algorithm and can predict the $K p, A E$ and $D s t$ indices only from their previous values with high accuracy and low time complexity.

\section{REFERENCES}

[1] Rolls, E.T., 1992. Neurophysiology and Functions of the Primate Amygdala. In: The Amygdala: Neurobiologycal Aspects of Emotion, Memory and Mental Dysfunction,

[2] McGaugh, J.L., F. Bermudez-Rattoni and R.A. Prado-Alcala, 1995. Plasticity in the Central Nervous System: Learning and Memory. Lawrence Erlbaum Associates, Inc., Mahwah, NJ., USA., pp: 17-39.

[3] Le Doux, J., 1996. The Emotional Brain. Simon and Schuster, New York, pp: 384.

[4] Morén, J. and C. Balkenius, 2000. A Computational Model of Emotional Learning in the Amygdala. In: From Animals to Animats 6: Proceedings of the 6th International Conference on the Simulation of Adaptive Behaviour, Meyer, J.A., A. Berthoz, D. Floreano, H.L. Roitblat and S.W. Wilson (Eds.). MIT Press, Cambridge, MA., USA., pp: 115-124.

[5] Balkenius, C., 2002. Emotion and learning-a computational model of the Amygdala. Ph.D. Thesis, Department of Cognitive Science, Lund University, Lund, Sweden.

[6] Balkenius, C., Morén J., 2001. Emotional Learning: A Computational Model of Amygdala, Cybernetics and Systems, Vol 32; Part 6, pp: 611636.

[7] Lucas, C., Shahmirzadi, D., and Sheikholeslami, N., 2004. Introducing BELBIC: Brain emotional learning based intelligent controller. Int. J. Intell. Autom. Soft Comput. 10: 11-21.

[8] Lucas, C., A. Abbaspour, A. Gholipour, B.N. Araabi and M. Fatourechi, 2003. Enhancing the performance of neurofuzzy predictors by emotional learning algorithm. Int. J. Informatica, 27 2, pp: 137-145.

[9] Lucas, C., R.M. Milasi and B.N. Araabi, 2006. Intelligent modeling and control of washing machine using Locally Linear Neuro-Fuzzy (LLNF). Asian J. Control, 8: 393-400. DOI: 10.1111/j.1934-6093.2006.tb00290.x

[10] Chandra M., 2005. Analytical Study of A Control Algorithm Based on Emotional Processing, M.S. Dissertation, Indian Institute of Technology Kanpur.

[11] Mehrabian, A. R., and Lucas, C., 2006a. Emotional Learning based Intelligent Robust Adaptive Controller for Stable Uncertain Nonlinear Systems, International Journal of Engineering and Mathematical Sciences, 2:4.
[12] Mehrabian, A. R., Lucas, C., Jafar Roshanian, 2006b. Aerospace launch vehicle control: an intelligent adaptive approach, Aerospace Science and Technology, 10, pp: 149-155, doi:10.1016/j.ast.2005.11.002

[13] Rouhani, H., M. Jalili, B.N. Araabi, W. Eppler and C. Lucas, 2007. Brain emotional learning based intelligent controller applied to neurofuzzy model of micro-heat exchanger. Expert Syst. Appl., 32: 911918. DOI: 10.1016/j.eswa.2006.01.047.

[14] Samadi, M.; Afzali-Kusha, A.; Lucas, C., 2007. Power management by brain emotional learning algorithm, 22-25 Oct., Guilin, pp: $78-81$. DOI: 10.1109/ICASIC.2007.4415571.

[15] Jafarzadeh, S., 2008. Designing PID and BELBIC Controllers in Path Tracking Problem, Int. J. of Computers, Communications \& Control, ISSN 1841-9836, E-ISSN 1841-9844, Vol. III (2008), Suppl. issue: Proceedings of ICCCC 2008, pp. 343-348.

[16] Beheshti, Z. and S.Z.M. Hashim, 2010. A review of emotional learning and it's utilization in control engineering. Int. J. Adv. Soft Comput. Appl., 2, pp: 191-208.

[17] Daryabeigi, E.; Markadeh, G.R.A.; Lucas, C., 2010. Emotional controller (BELBIC) for electric drives - A review, 7-10 Nov., Glendale, AZ, pp: 2901 - 2907. 10.1109/IECON.2010.5674934

[18] Lucas, C. 2010. BELBIC and Its Industrial Applications: Towards Embedded Neuroemotional Control Codesign, Integrated Systems, Design and Technology 2010, Part 3, 203-214, DOI: 10.1007/978-3642-17384-4_17

[19] Gholipour, A., C. Lucas and D. Shahmirzadi, 2004. Predicting geomagnetic activity index by brain emotional learning. WSEAS Trans. Syst., 3m pp: 296-299.

[20] Babaie, T., Karimizandi, Lucas, 2008. Learning based brain emotional intelligence as a new aspect for development of an alarm system, Soft Comput. 12, pp:857-873.

[21] Mirmomeni M., et al., 2010a. Introducing adaptive neurofuzzy modeling with online learning method for prediction of time-varying solar and geomagnetic activity indices, doi:10.1016/j.eswa.2010.05.059

[22] Mirmomeni, M., E. Kamaliha, S. Parsapoor and C. Lucas, 2010b. Variation of embedding dimension as one of the chaotic characteristics of solar and geomagnetic activity indices. National Academy of Sciences of the Republic of Armenia, pp: 338-349.

[23] Bala, R., P. H. Reiff, and J. E. Landivar, 2009. Real-time prediction of magnetospheric activity using the Boyle Index, Space Weather, 7, S04003, doi:10.1029/2008SW000407.

[24] Emery B. A., et al, 2009. Solar wind structure sources and periodicities of auroral electron power over three solar cycles, Journal of Atmospheric and Solar-Terrestrial Physics 71 1157-1175 doi:10.1016/j.jastp.2008.08.005

[25] Troshichev, O., Sormakov, D., Janzhura, A., 2010. Relation of PC index to the geomagnetic storm Dst variation, Journal of Atmospheric and Solar-Terrestrial Physics, doi:10.1016/j.jastp.2010.12.015

[26] Cerrato, Y., Saiz, E., Cid, C., Gonzalez, W. D., Palacios, J., 2011. Solar and interplanetary triggers of the largest Dst variations of the solar cycle 23. Journal of Atmospheric and Solar-Terrestrial Physics, doi:10.1016/j.jastp.2011.09.001.

[27] Jang, J.-S.R., 1993. ANFIS: adaptive-network-based fuzzy inference system, IEEE Transactions on Systems, Man and Cybernetics, 23 (3), pp: 665 - 685, doi: $10.1109 / 21.256541$ 